\title{
Enforcing Passivity for Admittance Matrices Approximated by Rational Functions
}

\author{
Bjørn Gustavsen, Member, IEEE and Adam Semlyen, Life Fellow, IEEE
}

\begin{abstract}
A linear power system component can be included in a transient simulation as a terminal equivalent by approximating its admittance matrix $Y$ by rational functions in the frequency domain. Physical behavior of the resulting model entails that it should absorb active power for any set of applied voltages, at any frequency. This requires the real part of $Y$ to be positive definite (PD). We calculate a correction to the rational approximation of $Y$ which enforces the PD-criterion to be satisfied. The correction is minimal with respect to the fitting error. The method is based on linearization and constrained minimization by Quadratic Programming. Examples show that models not satisfying the PD-criterion can lead to an unstable simulation, even though the rational approximation has stable poles only. Enforcement of the PD-criterion is demonstrated to give a stable result.
\end{abstract}

\section{INTRODUCTION}

$\mathbf{T}$ HIS paper deals with the topic of including in an electromagnetic transient simulation an admittance matrix $Y$ whose elements have been approximated ("fitted") in the frequency domain by rational functions. Examples of applications are the modeling of power transformers at high frequencies [1], [2] and the modeling of network equivalents [3].

Past experience has shown that simulations involving a fitted $Y$ can sometimes lead to an unstable simulation, even though the elements of $Y$ have been fitted using stable poles only.

A useful mitigation technique is to require the network to be passive, i.e., the component must absorb active power for any set of applied voltages, at any frequency. The resulting equivalent may still have unphysical electrical components, but the probability of an unstable simulation becomes greatly reduced.

A simple method for enforcing passivity has been introduced in [2]. However, then the criterion for assessing passivity was not sufficiently conservative as the focus was on the eigenvalues of $Y$ instead of the eigenvalues of $G=\operatorname{Re}\{Y\}$. Also, the correction made to the original fitting was constant over a wide frequency range and thus not minimal.

This paper presents a new method for enforcing passive behavior. Using the original fitting as a starting point in an iterative procedure, a constrained least squares fitting is calculated where the constraints are directly related to the criterion for passivity. As a result, the correction made to the original fitting is minimal in terms of the resulting fitting error.

Manuscript received October 6, 1999. This work was supported in part by the Natural Sciences and Engineering Research Council of Canada.

B. Gustavsen is currently on leave at the University of Toronto. He is with SINTEF Energy Research, N-7465 Trondheim, Norway (e-mail: bjorn.gustavsen@energy.sintef.no)

A. Semlyen is with the Department of Electrical and Computer Engineering, University of Toronto, Toronto, Ontario, Canada M5S 3G4 (e-mail: semlyen@ecf.utoronto.ca).

Publisher Item Identifier S 0885-8950(01)02312-4.

\section{CRITERION FOR PASSIVITY}

We are given in the frequency domain a component defined by an admittance matrix $Y$ :

$$
i=Y v
$$

For any complex vector $v$ we get for the absorbed power

$$
P=\operatorname{Re}\left\{v^{*} Y v\right\}=\operatorname{Re}\left\{v^{*}(G+j B) v\right\}=\operatorname{Re}\left\{v^{*} G v\right\}
$$

where the asterisk $*$ denotes transpose and conjugate. It follows that $P$ will always be positive only if all eigenvalues of $G$ are positive. Thus, a criterion for passivity is that $G=\operatorname{Re}\{Y\}$ be positive definite (PD).

We note that $G$ is a symmetric, real matrix. Therefore, all eigenvalues of $G$ are real.

\section{ENFORCEMENT OF PASSIVITY}

It is assumed that the rational approximation of $Y$ has been calculated with a fairly high accuracy. This makes it possible to force any negative eigenvalue of $G(\omega)$ to be positive by making only a small correction to the rational approximation. This justifies an approach based on linearization.

\section{A. Initial Solution}

We are given a matrix $Y$ whose elements $i, j$ have been approximated by a rational function of the form

$$
Y_{\text {fit }_{i, j}}(s)=\sum_{m=1}^{N} \frac{c_{m}}{s-a_{m}}+d+s e .
$$

Our task is to modify all or some of the parameters $c_{m}, a_{m}, d$ and $e$ for the elements of $Y_{\text {fit }}$ so that all eigenvalues of $G_{\text {fit }}=$ $\operatorname{Re}\left\{Y_{\text {fit }}\right\}$ become positive.

\section{B. Linearization}

The selected parameters of the rational approximation $Y_{\text {fit }}$ are placed in a single vector $x$, and the columns of $Y_{\text {fit }}$ are placed in a vector $y_{\text {fit }}$. Linearization of (3) gives the following incremental relation between $x$ and $y_{\text {fit }}$ :

$$
\Delta y_{\text {fit }}=M \Delta x
$$

Details of the calculation of $M$ are given in Appendix A.

Thus, a linear relation results between $x$ and $g_{\mathrm{fit}}$ :

$$
\Delta g_{\text {fit }}=\operatorname{Re}\{M\} \Delta x=P \Delta x
$$

where vector $g_{\text {fit }}$ contains the elements of $G_{\text {fit }}$. 
Finally, a linear relation is calculated between $g_{\mathrm{fit}}$ and the eigenvalues $\lambda$ of $G_{\mathrm{fit}}$ :

$$
\Delta \lambda=Q \Delta g_{\mathrm{fit}} .
$$

Combining (5) and (6) gives a linearized relation between the eigenvalues of $G_{\text {fit }}$ and the parameter vector $x$ :

$$
\Delta \lambda=Q P \Delta x=R \Delta x .
$$

Appendix B gives details regarding the calculation of $Q$.

\section{Constrained Least Squares Approximation}

We want to find a least squares solution $y_{\text {fit }}(x, s)$ to the problem

$$
y(s)-y_{\mathrm{fit}}(x, s) \rightarrow 0
$$

subject to passivity constraints for $g_{\text {fit }}$. Linearization using (4) gives

$$
y(s)-\left(y_{\text {fit }}^{0}(s, x)+M \Delta x\right) \rightarrow 0 .
$$

We require the eigenvalues $\lambda$ of $G_{\text {fit }}$ to be positive. This is achieved by (7):

$$
\Delta \lambda=R \Delta x \geq-\lambda .
$$

Equation (9) and (10) can be rewritten in the standard form:

$$
\begin{aligned}
& A \Delta x \rightarrow b \\
& B \Delta x \leq c
\end{aligned}
$$

where

$$
A=M, \quad b=y-y_{\text {fit }}^{0}, \quad B=-R, \quad c=\lambda .
$$

A least squares solution for (11) is calculated using Quadratic Programming $(\mathrm{QP})$ :

minimize

$$
\frac{1}{2} \Delta x^{T} H \Delta x-f^{T} \Delta x
$$

subject to

$$
B \Delta x \leq c
$$

where

$$
H=A^{T} A, \quad f=A^{T} b .
$$

\section{Preprocessing}

We have found it useful to separately enforce positive definiteness for $D$ and $E$ of the rational approximation $[D$ and $E$ are composed of $d$ and $e$ in (3)]. This is achieved as follows:

1) Diagonalize $D$ and $E$ :

$$
D=T_{D} \Lambda_{D} T_{D}^{-1}, \quad E=T_{E} \Lambda_{E} T_{E}^{-1} .
$$

Negative eigenvalues in $\Lambda_{D}$ and $\Lambda_{E}$ are set equal to 0 and modified matrices $D_{\mathrm{mod}}$ and $E_{\mathrm{mod}}$ are calculated by (15) ( $D_{\text {mod }}$ and $E_{\text {mod }}$ have no negative eigenvalues.)

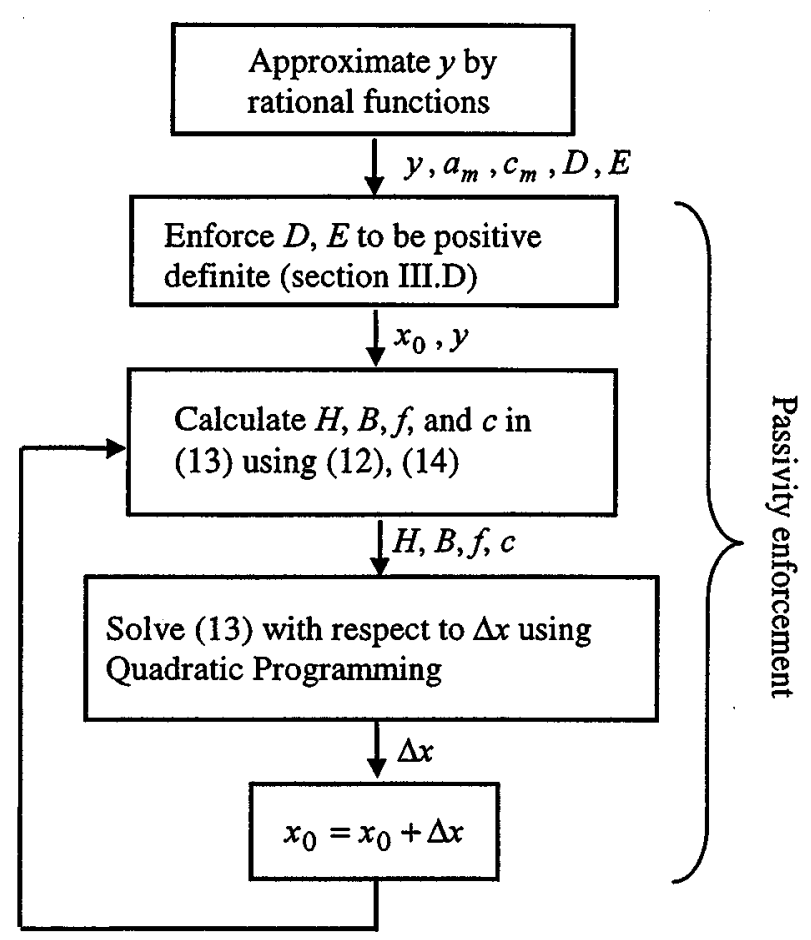

Fig. 1. Overview of calculation.

2) $D_{\text {mod }}$ and $s E_{\text {mod }}$ are subtracted from the data ( $\left.Y_{\text {meas }}\right)$, which is subjected to a new fitting with $D$, and $E$ restricted to be zero. Finally, $D_{\text {mod }}$ and $E_{\text {mod }}$ are added to the new rational approximation.

Note that $G_{\text {fit }}(s)$ does not depend on $E$, but negative eigenvalues in $E$ may lead to an unstable simulation and are therefore not tolerated.

\section{E. Program Overview}

Fig. 1 gives an overview of the calculation procedure. The program takes as input the original data $y$, and the parameters of the rational approximation. $D$ and $E$ are first enforced to be positive definite. The parameters of the rational approximation are placed in $x_{0}$ and a correction $\Delta x$ is calculated using Quadratic Programming and added to $x_{0}$. The procedure is repeated until all constraints have been satisfied. The linearizations (4) and (7) are recalculated in each iteration.

\section{PRACTICAL CONSIDERATIONS}

\section{A. Passivity Outside Fitting Range}

In general, $Y$ will have been fitted within a certain frequency range $\left(\omega_{1}-\omega_{2}\right)$. In order to ensure $G_{\text {fit }}(\omega)$ to be positive definite also outside this frequency range, we include additional frequency samples when building the constraint matrices $B$ and $c$ of the QP-problem (13).

\section{B. Updating of QP-Problem During Iterations}

Iterations as indicated in Fig. 1 are in general needed because (13) represents a linear model of a nonlinear problem. In our implementation we use only residues $\left[c_{m}\right.$ in (3)] as parameters in $x$ (see Section IV-C below). This results in (3) being linear 
in $x$, and thus $H$ in (13) will not change during the iterations. The other matrices $(B, f, c)$ need to be recalculated during the iterations.

\section{Reduction of Problem Size}

The number of elements in $x$ is reduced by nearly a factor of 4 as follows:

- Only residues are kept as unknowns (poles as well as elements of $D, E$ are considered known).

- Redundant elements occurring in $x$ due to symmetry of $Y$ are removed.

Furthermore, the number of rows in $B$ and $c$ can be drastically reduced by including only rows where a constraint violation occurs. When doing iterations as in Fig. 1, it is then necessary to also include in $B$ rows where violations occurred in previous steps (since violations may reappear).

\section{Sparsity}

Several of the matrices used in the building of the QP-problem (13) are sparse. This is the case for matrices $M, P$, and $A$. When calculating $H, B$, and $f$ via these matrices we operate directly on the nonzero elements, thus increasing computational efficiency and avoiding the need to store zero elements.

We note that in the QP-problem $B$ is full, whereas $H$ is blockdiagonal with as many blocks as there are elements in $Y$.

\section{Sequential Solution}

The measures mentioned in Section IV-C make it possible to solve relatively large systems. Using Matlab, problem sizes with up to 250 elements in $x$ are readily handled. However, larger problem sizes can occur, for instance in the case of network equivalents where very high order fittings may be used. A further reduction of $x$ is possible by redoing the iterations in Fig. 1 with different partitions of $x$.

We have implemented a simple method for partitioning $x$ which we refer to as sequential solution. The method can be outlined as follows:

1) Sort the elements of $x$ so that the magnitudes of the associated poles come in ascending order.

2) In each iteration in Fig. 1, use a contiguous set of elements from $x$. The sets of elements between two successive iterations are overlapping. Include in $B$ only rows corresponding to the frequencies covered by the poles of the partition.

3) Repeat the iterations in Fig. 1 until all elements in $x$ have been utilized.

The rationale of this procedure is the following: when a negative eigenvalue occurs at a given frequency $\omega_{0}$, partial fractions in (3) whose poles have magnitude in the neighborhood of $\omega_{0}$ are believed to be good candidates for enforcing the eigenvalue to become positive.

\section{Calculated Results}

In this section we show numerical results demonstrating the method of passivity enforcement and its effects on a time

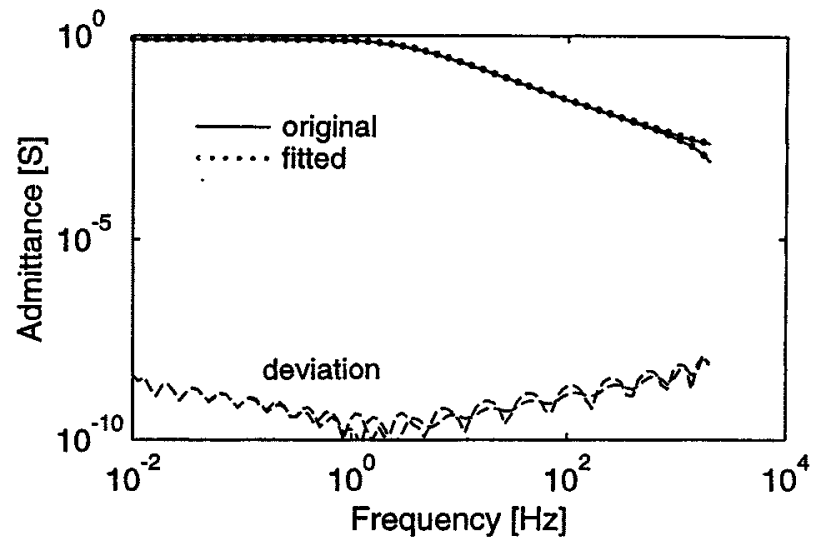

Fig. 2. Elements of $Y$ for single phase overhead line.

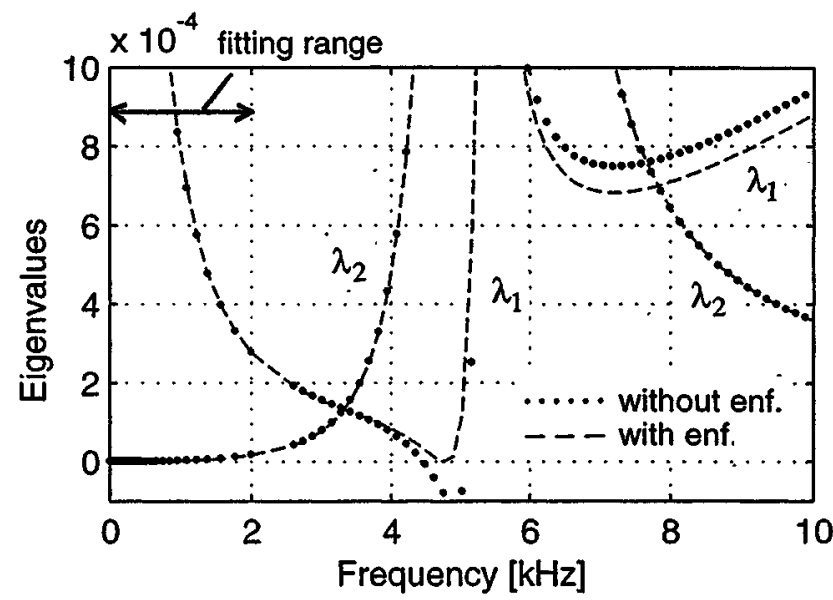

Fig. 3. Enforcing eigenvalues of $G_{\text {fit }}$ to become positive.

domain simulation. The initial rational approximation is in all examples calculated using Vector Fitting [4] and the time domain simulations are based on trapezoidal integration, similarly as in EMTP.

\section{A. Exact PI-Equivalent for Transmission Line}

In this example we model a $25 \mathrm{~km}$ single phase overhead line as an exact PI-equivalent (admittance matrix).

Fig. 2 shows the phase domain elements of the 2 by $2 Y$-matrix as fitted by 30 poles in the range $0.01 \mathrm{~Hz}-2000 \mathrm{~Hz}$. We note that all (four) elements have been fitted to an accuracy better than $10^{-8}$ within the fitting range.

One eigenvalue of $G_{\mathrm{fit}}=\operatorname{Re}\left\{Y_{\mathrm{fit}}\right\}$ is negative around $5 \mathrm{kHz}$, implying that $Y_{\text {fit }}$ is not passive. Passivity was enforced by modifying the residues $c_{m}$ of $Y_{\text {fit }}$ using the QP-approach of Fig. 1. Fig. 3 shows the eigenvalues of $G_{\text {fit }}$, before and after passivity enforcement. (A single iteration was used). We note that all eigenvalues have become positive, at all frequencies. The passivity enforcement had the effect of increasing the RMS-error in the fitting range from $2.48 \mathrm{E}-9$ to $3.76 \mathrm{E}-8$, which is still very small.

We now demonstrate the effect of passivity enforcement on a time domain simulation. The transmission line (Fig. 4) is 
open at $\mathrm{t}=10 \mathrm{~ms}$

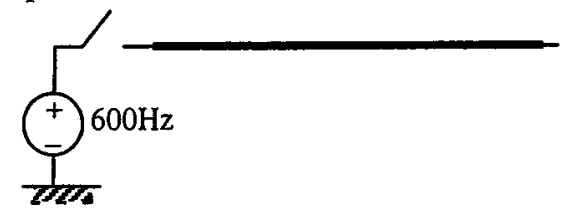

Fig. 4. Disconnecting a transmission line.

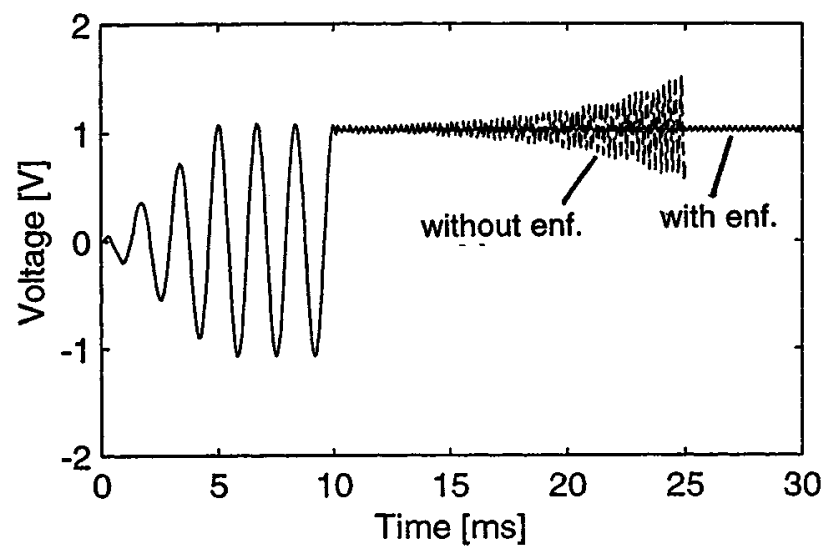

Fig. 5. Effect of passivity enforcement on a time domain simulation.

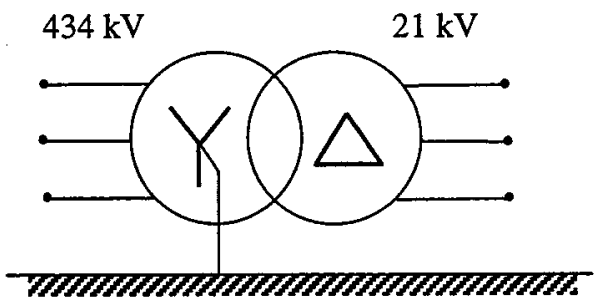

Fig. 6. 410 MVA generator step-up transformer.

energized by a $600 \mathrm{~Hz}$ voltage source which is ramped up from 0 to $1 \mathrm{~V}$ in $5 \mathrm{~ms}$. At $t=10 \mathrm{~ms}$ the line is disconnected from the source.

Fig. 5 shows the simulated voltage on the open line end. Without passivity enforcement the voltage is seen to become unstable after disconnecting the line. The state equation realization of the disconnected line was found to have one unstable pair of poles (302 $\pm j 30919)$, which resonates at $4919 \mathrm{~Hz}$. Passivity enforcement moved the pole pair to $(-25 \pm j 30463)$ which is stable, although weakly damped. Fig. 5 shows that a stable simulation has been achieved.

\section{B. Transformer Model}

We return to the transformer measurements used in [2] for which the admittance matrix $Y$ has been measured in the frequency range $1 \mathrm{kHz}-200 \mathrm{kHz}$ [5].

The measurements were fitted using a 10th order approximation. Fig. 7 shows the eigenvalues of $G=\operatorname{Re}\{Y\}$ in the range $1 \mathrm{kHz}-200 \mathrm{kHz}$, and the eigenvalues of $G_{\mathrm{fit}}=\operatorname{Re}\left\{Y_{\mathrm{fit}}\right\}$ in the range $0 \mathrm{~Hz}-300 \mathrm{kHz}$. Fig. 8 shows an expanded view of Fig. 7. It is seen that $G$ and $G_{\mathrm{fit}}$ have negative eigenvalues below $10 \mathrm{kHz}$.

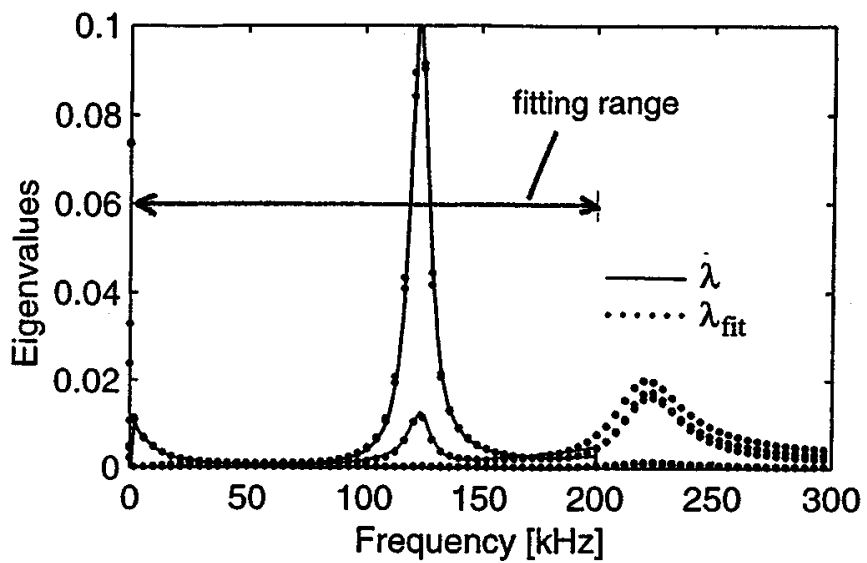

Fig. 7. Eigenvalues of $G$ and $G_{\text {fit }}$.

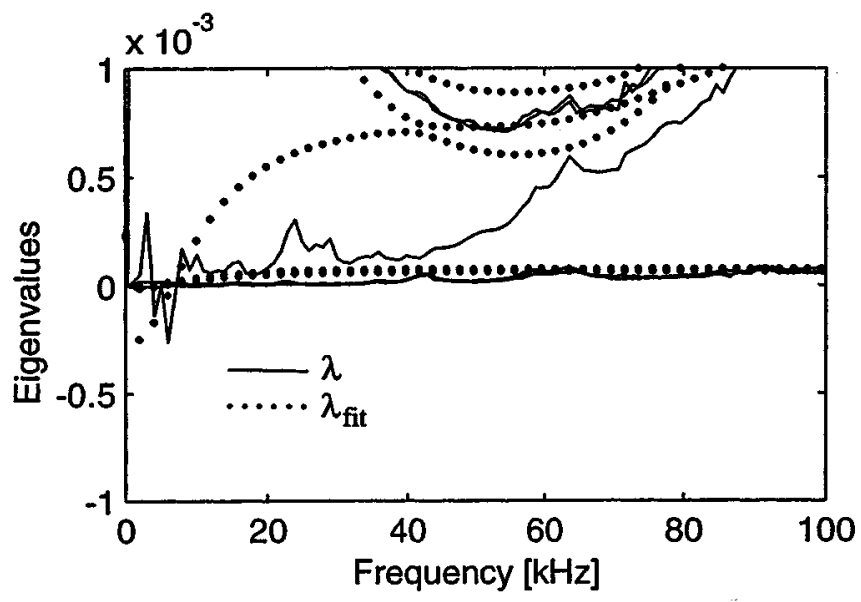

Fig. 8. Expanded view of Fig. 7.

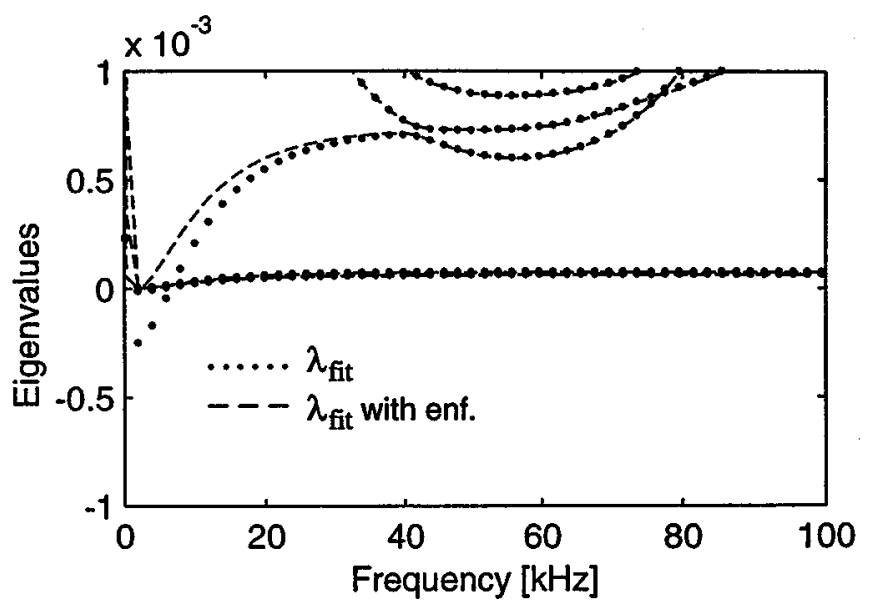

Fig. 9. Enforcing eigenvalues of $G_{\mathrm{fit}}$ to become positive.

The QP-approach in Fig. 1 enforced $G_{\text {fit }}$ to become passive in a single iteration. Fig. 9 shows the eigenvalues of $G_{\text {fit }}$ before and after passivity enforcement. It is seen that the negative eigenvalues have been forced to be positive. The rms-error of the fitting of $Y$ (between $1 \mathrm{kHz}$ and $200 \mathrm{kHz}$ ) increased from $1.894 \mathrm{E}-4$ to $1.897 \mathrm{E}-4$. 


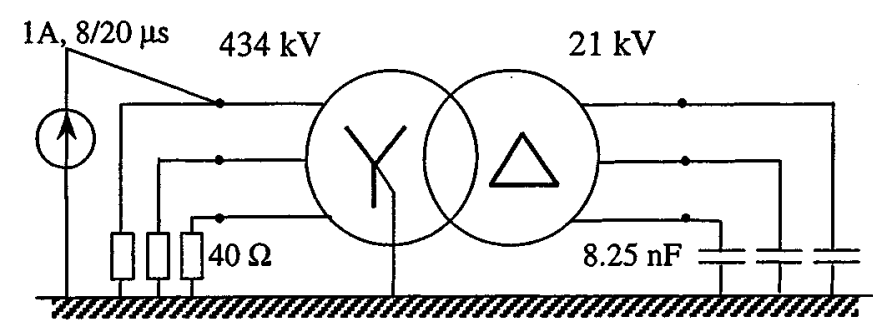

Fig. 10. Voltage transfer calculation.

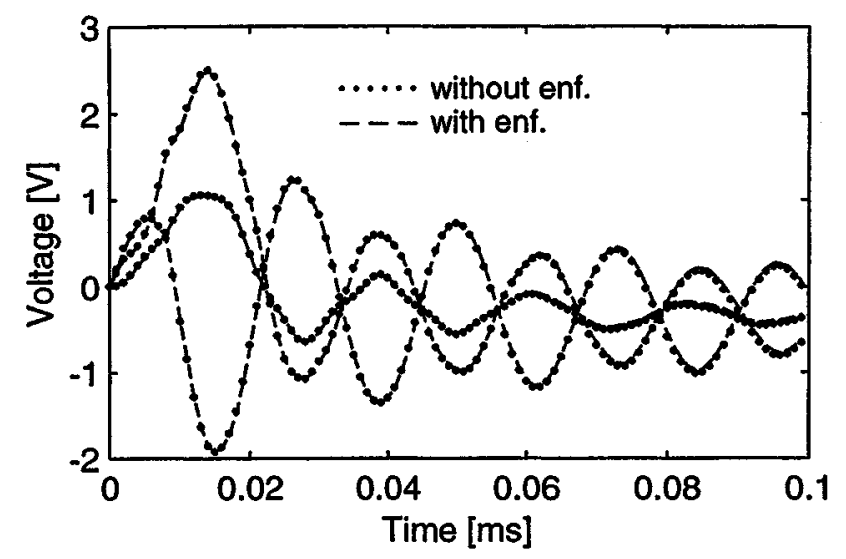

Fig. 11. Voltage at low-voltage terminals: $0-0.1 \mathrm{~ms}$.

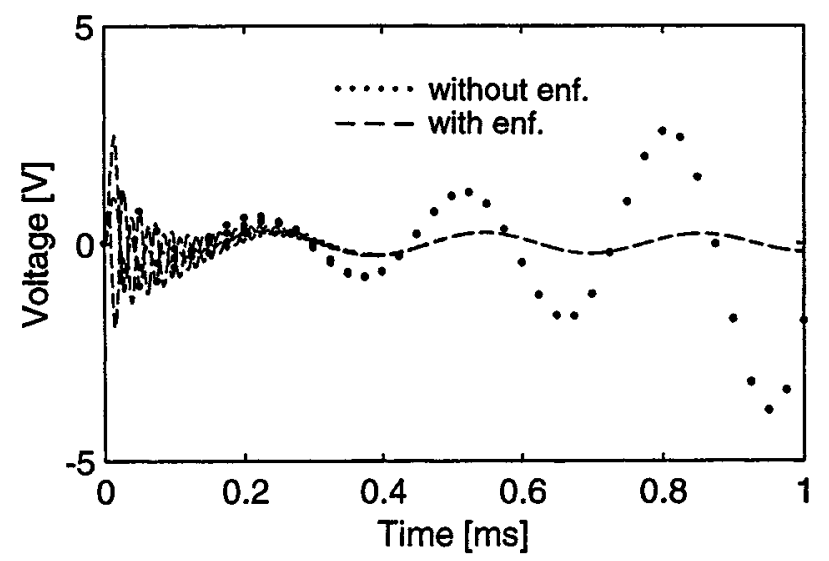

Fig. 12. Voltage at low-voltage terminals: $0-1 \mathrm{~ms}$.

The significance of passivity enforcement will be demonstrated by an example of voltage transfer calculation, as shown in Fig. 10. The circuit represents the situation that the transformer is connected to very long cables at the high-voltage side, and very short cables $(\sim 25 \mathrm{~m})$ at the low-voltage side. An incident $8 / 20 \mu$ s wave impinges the transformer at one of the high voltage terminals.

Fig. 11 shows the effect of the passivity enforcement on the simulated voltages at the low-voltage terminals, for the time frame $0-0.1 \mathrm{~ms}$. It is seen that the passivity enforcement has only a very small effect on the simulated waveforms.

Fig. 12 shows the simulated waveforms in the time frame $0-1 \mathrm{~ms}$. It is seen that the passivity enforcement has the effect of mitigating a zero sequence oscillation with negative damping.

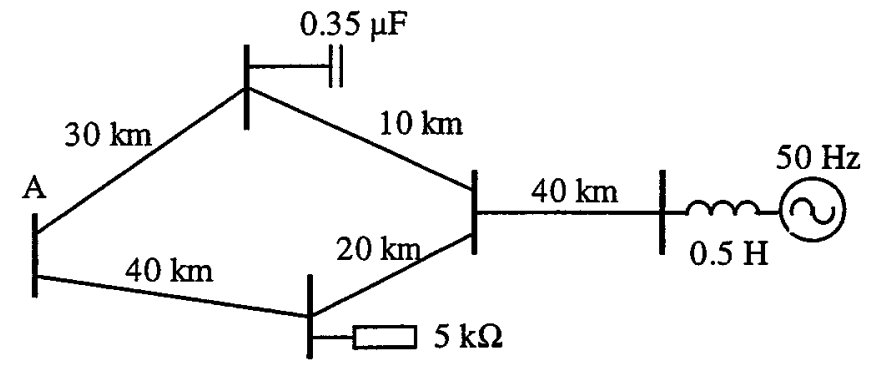

Fig. 13. $300 \mathrm{kV}$ network.

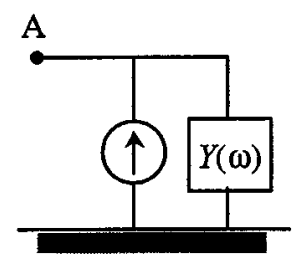

Fig. 14. Network equivalent for bus A.

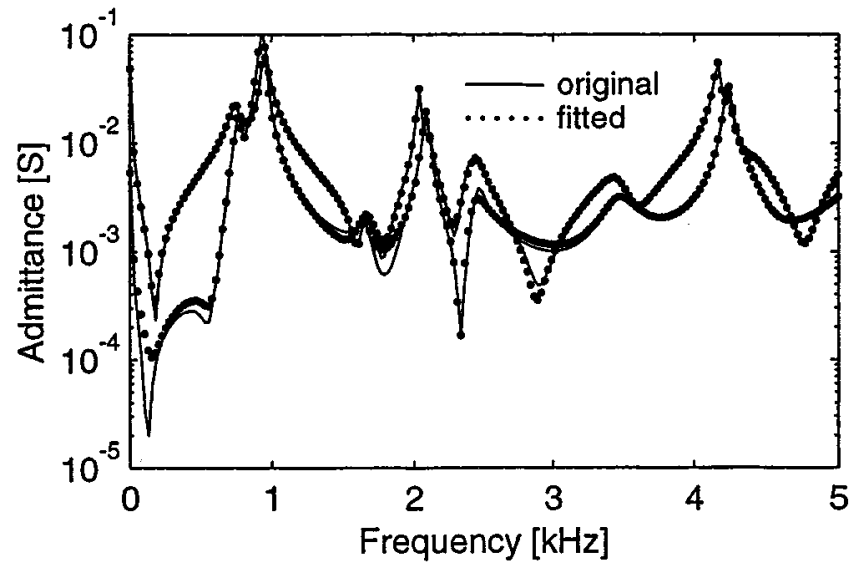

Fig. 15. Elements $Y(1,1)$ and $Y(2,1)$.

\section{Network Equivalent}

Network equivalents for parts of a network may be used as a means to reduce the computation time of time domain simulations [3]. However, unstable simulations may result when passivity is not enforced.

Fig. 13 shows a simple $300 \mathrm{kV}$ system where the overhead lines are untransposed. A network equivalent for the system was first calculated in the phase domain with respect to bus A, as shown in Fig. 14.

The elements of the 3 by 3 admittance $Y(\omega)$ were fitted in the range $5 \mathrm{~Hz}-5 \mathrm{kHz}$ using a common set of 26 poles. Fig. 15 shows the elements $Y(1,1)$ and $Y(2,1)$.

Negative eigenvalues for $G$ were observed at about $1.7 \mathrm{kHz}$. Fig. 16 shows the effect of passivity enforcement on the eigenvalues, in the range $1 \mathrm{kHz}-3 \mathrm{kHz}$. It is seen that the eigenvalues become positive after enforcement of passivity.

The network equivalent in Fig. 14 was represented by an electrical network in ATP-EMTP. A simulation of the equivalent in open circuit was performed in ATP, starting from a phasor 


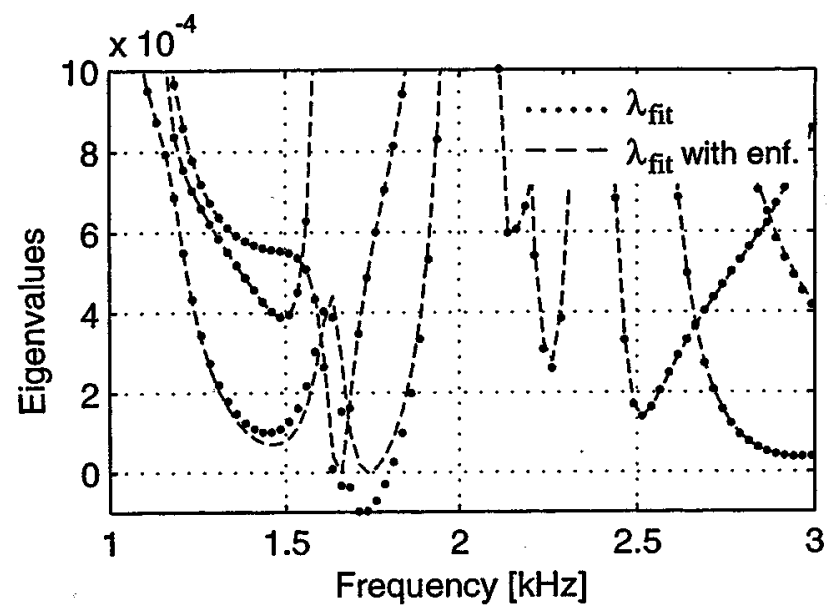

Fig. 16. Effect of passivity enforcement on eigenvalues of $G$.

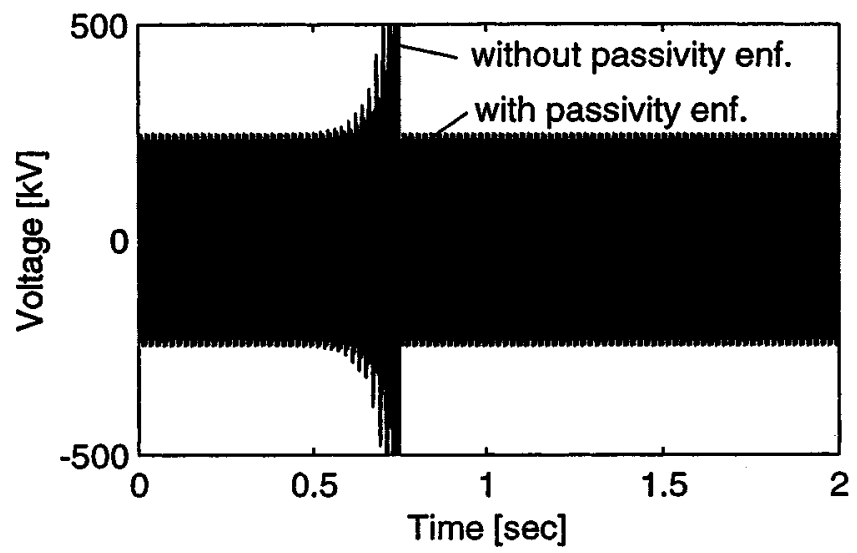

Fig. 17. Time domain simulation.

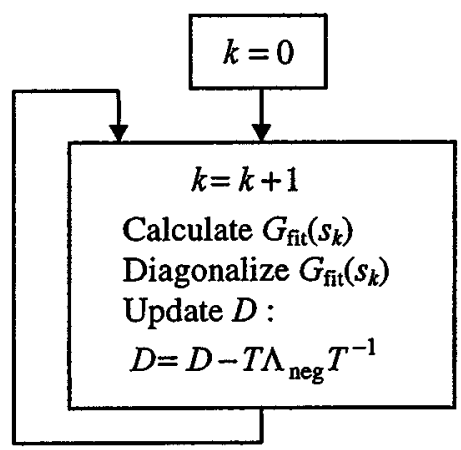

Fig. 18. Enforcing passivity by simplistic approach.

solution as initial condition. Fig. 17 shows the node voltage at bus $\mathrm{A}$, before and after enforcement of passivity. It is seen that the passivity enforcement mitigates an unstable simulation. A further analysis showed that without passivity enforcement, the circuit had two unstable eigenvalues

$$
\lambda_{1,2}=24.37 \pm j 11032 .
$$

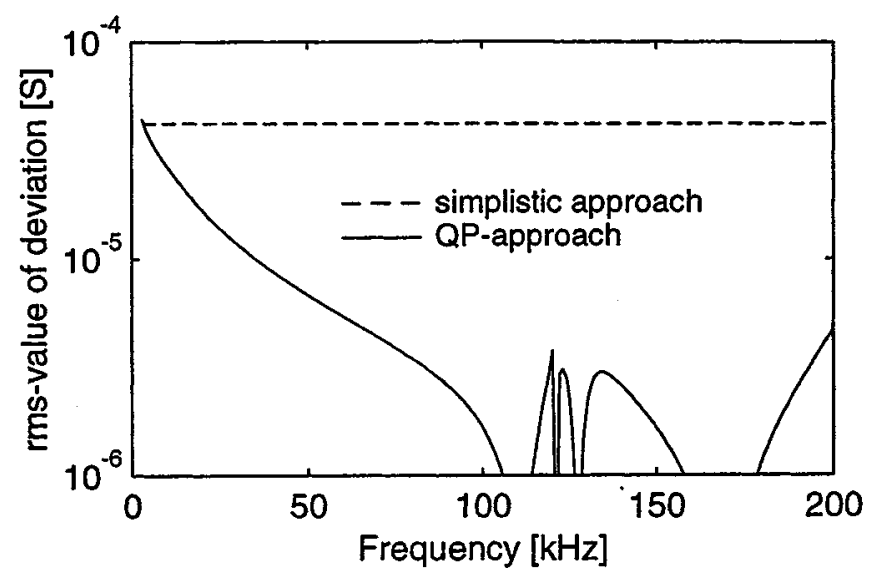

Fig. 19. Change in $Y_{\text {fit }}$ due to passivity enforcement.

\section{SIMPLISTIC APPROACH}

In the following we show a simple approach for passivity enforcement, based on the method developed in [2]. This approach is very easy to implement in a computer program, but gives a larger correction to the fitted $Y$, as compared to the previously described QP-approach.

The real part of the rational approximation (3) can be written as:

$$
G_{\mathrm{fit}_{i, j}}(s)=d+\operatorname{Re}\left\{\sum_{m=1}^{N} \frac{c_{m}}{s-a_{m}}\right\}=d+p(s) .
$$

The full matrix becomes

$$
G_{\text {fit }}(s)=D+P(s) .
$$

We consider a single frequency s and diagonalize $G_{\mathrm{fit}}$ :

$$
T \Lambda T^{-1}=D+P
$$

where $\Lambda$ contains the eigenvalues. $\Lambda$ is separated into a sum:

$$
T\left(\Lambda_{\text {pos }}+\Lambda_{\text {neg }}\right) T^{-1}=D+P
$$

where $\Lambda_{\text {pos }}$ and $\Lambda_{\text {neg }}$ contain the positive and negative eigenvalues, respectively. Moving the second term of the left side to the right side produces a modified $G_{\mathrm{fit}}$ which is PD:

$$
G_{\text {fit, corr }}=T \Lambda_{\text {pos }} T^{-1}=D-T \Lambda_{\text {neg }} T^{-1}+P .
$$

Thus, $G_{\text {fit }}$ can be modified to have its negative eigenvalues replaced by zeros by adding a correction to $D$. This procedure is repeated for all frequencies for which we require passivity:

This alternative approach gives a correction to $Y_{\text {fit }}$ which is larger than the one obtained by the QP-approach. Fig. 19 compares the norm of the change to $Y_{\text {fit }}$ for the transformer example in the previous section, when using the simplistic approach and the QP-approach, respectively. Despite of the larger change to $Y_{\text {fit }}$ the time domain simulation (Fig. 11) was nearly identical for the two approaches.

In other cases the high accuracy of the QP-approach is crucial. Fig. 20 shows a situation of a trapped charge simulation for an exact PI-equivalent of a $25 \mathrm{~km} 3$ phase overhead line, whose 


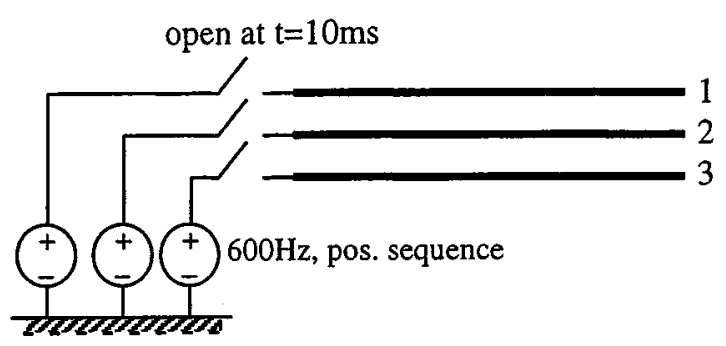

Fig. 20. Disconnecting a transmission line.

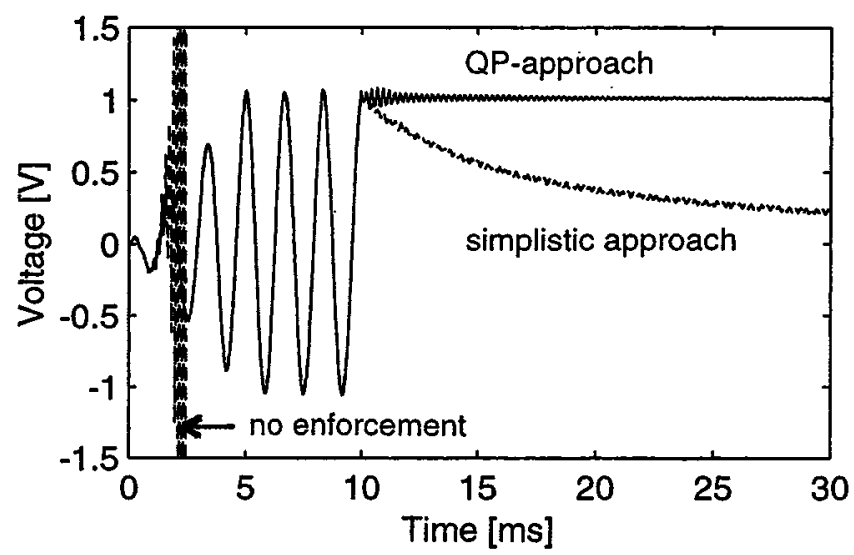

Fig. 21. Trapped charge simulation.

6 by $6 Y$ was fitted by 30 poles in the range $0.01 \mathrm{~Hz}-2000 \mathrm{~Hz}$. The transmission line (exact PI) is energized by a 3 phase $600 \mathrm{~Hz}$ voltage source which is ramped up from 0 to $1 \mathrm{~V}$ in $5 \mathrm{~ms}$. At $t=10 \mathrm{~ms}$ the line is disconnected from the source.

Fig. 21 shows the simulated voltage on conductor 1 at the receiving end. It is seen that without passivity enforcement the voltage blows up during ramp-up. When enforcing passivity using the simplified approach the trapped charge leaks out from the line. This result is incorrect as the shunt admittance matrix for the line had zero conductance. When enforcing passivity by the QP approach there is no discharge of the trapped charge (no drift was detected after $0.5 \mathrm{~s}$ ).

\section{CONCLUSION}

The paper has presented a new method for the enforcement of passivity for an admittance matrix $Y$ approximated by rational functions of the form given in (3). Passivity is ensured by enforcing the eigenvalues of $G_{\text {fit }}=\operatorname{Re}\left\{Y_{\text {fit }}\right\}$ to be positive.

The approach adopted is as follows:

1) Positive definiteness is first enforced for $D$ and $E$ of the rational approximation.

2) Positive definiteness is then enforced for $G_{\text {fit }}$ by calculating a correction to the residues of the rational approximation. The correction is based on linearization, leading to a constrained linear least squares problem which is solved by Quadratic Programming.
A sequential solution based on residue partitioning is used if the number of residues becomes too large for Quadratic Programming.

The main characteristics of the method are:

1) The correction is minimal in the sense that it ensures passivity with a smallest possible increase in the RMS-error of the rational approximation.

2) Iterations are in general needed due to the nonlinear nature of the problem. 1-3 iterations are normally sufficient.

The method was successfully applied to an exact PI-equivalent for an overhead line, a high frequency model of a power transformer, and to a network equivalent. In these examples the passivity enforcement had the effect of mitigating instability from the time domain simulation.

A simplified approach (Section VII) was also proposed for passivity enforcement. However, this approach leads to a larger change in $Y_{\text {fit }}$ within the fitting range.

\section{APPENDIX A \\ Calculation of $M$}

In this section we show how to build the matrix $M$ in the QP-approach.

For each element $(i, j)$ of $Y$ we have given a rational approximation

$$
Y_{\mathrm{fit}_{i, j}}(s)=\sum_{m=1}^{N} \frac{c_{i, j, m}}{s-a_{i, j, m}}+d_{i, j}+s e_{i, j} .
$$

For simplicity we now assume $Y_{\text {fit }}$ to be of dimension 2 by 2, and we also assume $N=2$ for the order of approximation. Stacking the columns of $Y_{\text {fit }}$ into a single column $y_{\text {fit }}$ and the corresponding residues into a column $x$, we obtain the linearized relationship between $y_{\text {fit }}$ and $x$

$$
\Delta y_{\text {fit }}=M \Delta x
$$

where, shown in the equation at the top of the next page.

Note that in our actual implementation we keep only the residues corresponding to the upper triangle of $Y$ as unknowns, as $Y$ is a symmetric matrix. Also, $M$ is never formulated in the form shown above since we operate directly on its nonzero elements for the calculation of $R$ in (7).

\section{APPENDIX B \\ Calculation of $Q$}

We consider the eigenproblem of $G_{\text {fit }}$ with focus on a particular eigenvalue $\lambda$ and its corresponding right eigenvector $v$ :

$$
\left(G_{\text {fit }}-\lambda I\right) v=0 .
$$

Linearization of (B.1) gives

$$
\left(G_{\text {fit }}-\lambda I\right) \Delta v+\left(\Delta G_{\text {fit }}-\Delta \lambda I\right) v=0 .
$$

Premultiplication of (B.2) with the corresponding left (row) eigenvector $w$ of $G_{\text {fit }}$ makes the first term in (B.2) disappear and we get

$$
\Delta \lambda=\frac{w \Delta G_{\mathrm{fit}} v}{w v}
$$




$$
M=\left[\begin{array}{cccccccc}
\frac{1}{s-a_{1,1,1}} & \frac{1}{s-a_{1,1,2}} & 0 & 0 & 0 & 0 & 0 & 0 \\
0 & 0 & \frac{1}{s-a_{2,1,1}} & \frac{1}{s-a_{2,1,2}} & 0 & 0 & 0 & 0 \\
0 & 0 & 0 & 0 & \frac{1}{s-a_{1,2,1}} & \frac{1}{s-a_{1,2,2}} & 0 & 0 \\
0 & 0 & 0 & 0 & 0 & 0 & \frac{1}{s-a_{2,2,1}} & \frac{1}{s-a_{2,2,2}}
\end{array}\right]
$$

Because $G_{\text {fit }}$ is symmetrical we have $w=v^{T}$, and when the eigenvectors have been normalized to unit length we get

$$
\Delta \lambda=v^{T} \Delta G_{\mathrm{fit}} v .
$$

Equation (B.4) is used only for distinct eigenvalues [6, p. 229] as it may produce an incorrect result for equal eigenvalues.

If two eigenvalues, $\lambda_{1}, \lambda_{2}$, are found to be closer than a certain $\varepsilon$, the sensitivities for these two eigenvalues are calculated by replacing $v$ in (B.4) by the matrix formed by (the arbitrary set within the two-dimensional null-space of) the two eigenvectors associated with the double eigenvalue $\lambda=\lambda_{1}=\lambda_{2}$. The resulting sensitivity in (B.4) now becomes a full (symmetric) $2 \times 2$ matrix

$$
\Delta \Lambda=\left[\begin{array}{cc}
\Delta \Lambda_{11} & \Delta \Lambda_{12} \\
\Delta \Lambda_{12} & \Delta \Lambda_{22}
\end{array}\right]
$$

The perturbations $\Delta \lambda_{1} \neq \Delta \lambda_{2}$ of the equal eigenvalues $\lambda_{1}$, $\lambda_{2}$ are the eigenvalues of $\Delta \Lambda$ of (B.5). They are nonlinear functions of $\Delta \Lambda_{11}, \Delta \Lambda_{22}, \Delta \Lambda_{12}$, but by putting $\Delta \Lambda_{12}=0$, we get

$$
\Delta \lambda_{1}=\Delta \Lambda_{11}, \quad \Delta \lambda_{2}=\Delta \Lambda_{22} .
$$

It now follows that the eigenpair $\lambda_{1}, \lambda_{2}$ is forced to be positive by the following constraints:

$$
\begin{aligned}
& \Delta \Lambda_{12}=0 \\
& \Delta \Lambda_{11} \geq-\lambda \\
& \Delta \Lambda_{22} \geq-\lambda .
\end{aligned}
$$

The above approach has been generalized for eigenvalues of higher multiplicity by replacing (B.7) by $\Delta \Lambda_{i j}=0$, and (B.8) by $\Delta \Lambda_{i i} \geq-\lambda$.

\section{ACKNOWLEDGMENT}

The authors would like to thank T. Ohnstad (Statnett SF, Norway) for allowing usage of data from the transformer measurements.

\section{REFERENCES}

[1] A. Morched, L. Marti, and J. Ottevangers, "A high frequency transformer model for the EMTP," IEEE Trans. PWRD, vol. 8, no. 3, pp. 1615-1626, July 1993.

[2] B. Gustavsen and A. Semlyen, "Application of vector fitting to the state equation representation of transformers for simulation of electromagnetic transients," IEEE Trans. PWRD, vol. 13, no. 3, pp. 834-842, July 1998.

[3] A. S. Morched, J. H. Ottevangers, and L. Marti, "Multi-port frequency dependent network equivalents for the EMTP," IEEE Trans. PWRD, vol. 8, no. 3, pp. 1402-1412, July 1993.

[4] B. Gustavsen and A. Semlyen, "Rational approximation of frequency domain responses by vector fitting," IEEE Trans. PWRD, vol. 14, no. 3, pp. 1052-1061, July 1999.

[5] T. Henriksen and S. Frydenlund, "Frequency characteristics of a 410 MVA two-winding three-phase transformer," Norwegian Electric Power Research Institute, TR F3923, Mar. 1992.

[6] D. K. Faddeev and V. N. Faddeeva, Computational Methods of Linear Algebra: W.H. Freeman and Company, 1963.

Bjørn Gustavsen was born in Norway, in 1965. He received the M.Sc. degree in 1989 and the Dr.Ing. degree in 1993, both from the Norwegian Institute of Technology. Since 1994, he has been working at SINTEF Energy Research (former EFI), mainly in the field of transient studies. He spent 1996 as a Visiting Researcher at the University of Toronto. In 1998, he implemented a phase domain line model in EMTDC, at the Manitoba HVDC Research Centre.

Adam Semlyen was born in 1923 in Rumania where he obtained the Dipl.Ing. degree and Ph.D. degree. He started his career there with an electric power utility and held academic positions at the Polytechnic Institute of Timisoara. In 1969, he joined the University of Toronto where he is a Professor in the Department of Electrical and Computer Engineering, emeritus since 1988. His research interests include steady state and dynamic analysis as well as computation of electromagnetic transients in power systems. 